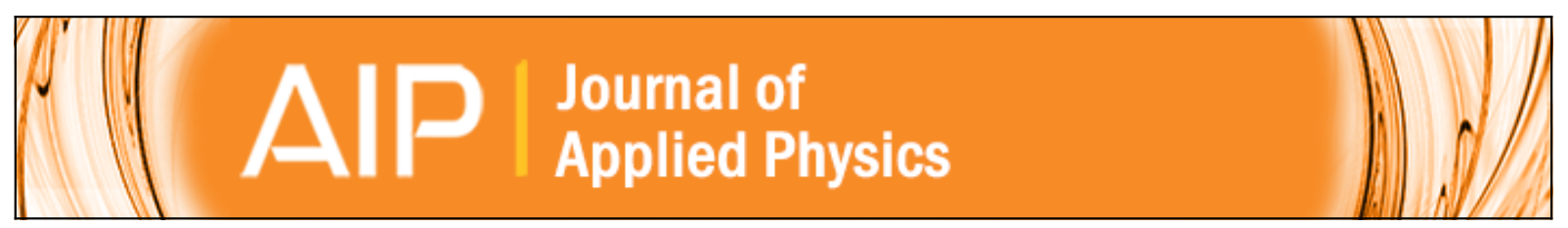

\title{
Optical power of semiconductor lasers with a low-dimensional active region
}

Levon V. Asryan and Zinaida N. Sokolova

Citation: Journal of Applied Physics 115, 023107 (2014); doi: 10.1063/1.4861408

View online: http://dx.doi.org/10.1063/1.4861408

View Table of Contents: http://scitation.aip.org/content/aip/journal/jap/115/2?ver=pdfcov

Published by the AIP Publishing

\section{Articles you may be interested in}

Influencing modulation properties of quantum-dot semiconductor lasers by carrier lifetime engineering Appl. Phys. Lett. 101, 131107 (2012); 10.1063/1.4754588

Role of carrier reservoirs on the slow phase recovery of quantum dot semiconductor optical amplifiers

Appl. Phys. Lett. 94, 041112 (2009); 10.1063/1.3073715

Ultralow noise optical pulse generation in an actively mode-locked quantum-dot semiconductor laser Appl. Phys. Lett. 88, 131106 (2006); 10.1063/1.2190443

Optically active erbium-oxygen complexes in GaAs

Appl. Phys. Lett. 84, 1683 (2004); 10.1063/1.1668323

Four-wave mixing mediated by the capture of electrons and holes in semiconductor quantum-well laser amplifiers Appl. Phys. Lett. 71, 3601 (1997); 10.1063/1.120453

MIT LINCOLN

LABORATORY CAREERS

Discover the satisfaction of innovation and service

to the nation
- Space Control

- Air \& Missile Defense

- Communications Systems \& Cyber Security

- Intelligence, Surveillance and

Reconnaissance Systems

- Advanced
Electronics
- Tactical Systems
- Homeland
Protection
- Air Traffic Control

LINCOLN LABORATORY

MassachusetTs Institute of TeChNOLOgY

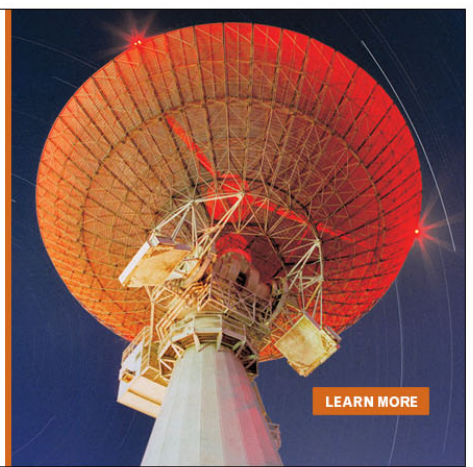




\title{
Optical power of semiconductor lasers with a low-dimensional active region
}

\author{
Levon V. Asryan ${ }^{1, a)}$ and Zinaida N. Sokolova, ${ }^{2, b}$ \\ ${ }^{1}$ Virginia Polytechnic Institute and State University, Blacksburg, Virginia 24061, USA \\ ${ }^{2}$ Ioffe Physico-Technical Institute, St. Petersburg 194021, Russia
}

(Received 28 November 2013; accepted 20 December 2013; published online 13 January 2014)

\begin{abstract}
A comprehensive analytical model for the operating characteristics of semiconductor lasers with a low-dimensional active region is developed. Particular emphasis is given to the effect of capture delay of both electrons and holes from a bulk optical confinement region into a quantum-confined active region and an extended set of rate equations is used. We derive a closed-form expression for the internal quantum efficiency as an explicit function of the injection current and parameters of a laser structure. Due to either electron or hole capture delay, the internal efficiency decreases with increasing injection current above the lasing threshold thus causing sublinearity of the light-current characteristic of a laser. (C) 2014 AIP Publishing LLC. [http://dx.doi.org/10.1063/1.4861408]
\end{abstract}

\section{INTRODUCTION}

A number of processes are involved in the operation of semiconductor lasers with a quantum-confined active region. ${ }^{1,2}$ Among them is the carrier capture from a bulk reservoir region [optical confinement layer (OCL)] into a low-dimensional active region. ${ }^{3-16}$ Analytical models can provide insights into these processes and be used to optimize the laser structures to achieve desired operating characteristics. To develop analytical approaches, certain assumptions and simplifications should be made. Reduction of the number of rate equations describing the carrier dynamics in a laser structure is one of such simplifications. Particularly, the use of rate equations for carriers of only one type can considerably simplify the analysis. This is commonly done in the literature-see, e.g., Refs. 14 and 15, in which just three rate equations were exploited (one for free carriers in the OCL, one for carriers confined in the active region, and one for photons) to study the laser power characteristics.

In this paper, we analyze the effect of both electron and hole capture delay from the OCL into a low-dimensional active region on the internal quantum efficiency $\eta_{\text {int }}$ and output optical power of semiconductor lasers. To describe such a general situation, we use rate equations both for electrons and holes. Remarkably, while the number of equations is now five (two for free electrons and holes in the OCL, two for electrons and holes confined in the active region, and one for photons), we are able to derive a closed-form expression for $\eta_{\text {int }}$ as an explicit function of the injection current, electron and hole capture velocities, and parameters of the laser structure.

\section{THEORETICAL MODEL}

While our model is general and applies to semiconductor lasers with a quantum-confined active region of any type [quantum wells (QWs), quantum wires, or quantum dots], we use here a QW laser to illustrate the model. We study the

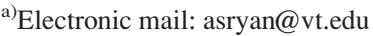

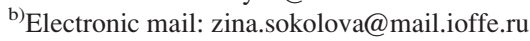

steady-state characteristics of the laser and, correspondingly, use the following set of steady-state rate equations:

for free electrons in the OCL $\left[b\left(\partial n^{\mathrm{OCL}} / \partial t\right)=0\right]$,

$$
\begin{aligned}
\frac{j}{e} & +N_{\mathrm{QW}} \frac{n^{\mathrm{QW}}}{\tau_{\mathrm{n}, \mathrm{esc}}}-N_{\mathrm{QW}} \mathrm{V}_{\mathrm{n}, \text { capt }, 0}\left(1-f_{\mathrm{n}}\right) n^{\mathrm{OCL}} \\
& -b B_{3 \mathrm{D}} n^{\mathrm{OCL}} p^{\mathrm{OCL}}=0,
\end{aligned}
$$

for free holes in the OCL $\left[b\left(\partial p^{\mathrm{OCL}} / \partial t\right)=0\right]$,

$$
\begin{aligned}
\frac{j}{e} & +N_{\mathrm{QW}} \frac{p^{\mathrm{QW}}}{\tau_{\mathrm{p}, \mathrm{esc}}}-N_{\mathrm{QW}} \mathrm{V}_{\mathrm{p}, \mathrm{capt}, 0}\left(1-f_{\mathrm{p}}\right) p^{\mathrm{OCL}} \\
& -b B_{3 \mathrm{D}} n^{\mathrm{OCL}} p^{\mathrm{OCL}}=0,
\end{aligned}
$$

for electrons confined in the QWs $\left(\partial n^{\mathrm{QW}} / \partial t=0\right)$,

$$
\begin{gathered}
\mathrm{v}_{\mathrm{n}, \text { capt }, 0}\left(1-f_{\mathrm{n}}\right) n^{\mathrm{OCL}}-\frac{n^{\mathrm{QW}}}{\tau_{\mathrm{n}, \mathrm{esc}}}-B_{2 \mathrm{D}} n^{\mathrm{QW}} p^{\mathrm{QW}} \\
-\mathrm{v}_{\mathrm{g}} g^{\max }\left(f_{\mathrm{n}}+f_{\mathrm{p}}-1\right) \frac{N}{S}=0,
\end{gathered}
$$

for holes confined in the QWs $\left(\partial p^{\mathrm{QW}} / \partial t=0\right)$,

$$
\begin{gathered}
\mathrm{v}_{\mathrm{p}, \text { capt }, 0}\left(1-f_{\mathrm{p}}\right) p^{\mathrm{OCL}}-\frac{p^{\mathrm{QW}}}{\tau_{\mathrm{p}, \mathrm{esc}}}-B_{2 \mathrm{D}} n^{\mathrm{QW}} p^{\mathrm{QW}} \\
-\mathrm{v}_{\mathrm{g}} g^{\max }\left(f_{\mathrm{n}}+f_{\mathrm{p}}-1\right) \frac{N}{S}=0,
\end{gathered}
$$

and for photons $(\partial N / \partial t=0)$,

$$
\mathrm{v}_{\mathrm{g}} N_{\mathrm{QW}} g^{\max }\left(f_{\mathrm{n}}+f_{\mathrm{p}}-1\right) N-\mathrm{v}_{\mathrm{g}}\left(\beta+\alpha_{\text {int }}\right) N=0 .
$$

We first list the quantities to be found from the solution of Eqs. (1)-(5): $n^{\mathrm{OCL}}$ and $p^{\mathrm{OCL}}$ are the free-electron and -hole densities in the OCL, $n^{\mathrm{QW}}$ and $p^{\mathrm{QW}}$ are the two-dimensional (2D) densities of electrons and holes confined in each of the QWs, and $N$ is the number of photons in the lasing mode. $f_{\mathrm{n}}$ is the occupancy of the lower edge of the electron 
quantum-confinement sub-band in the $\mathrm{QW}$ and $f_{\mathrm{p}}$ is the occupancy of the upper edge of the hole quantum-confinement sub-band in the QW. The occupancies $f_{\mathrm{n}}$ and $f_{\mathrm{p}}$ are not independent quantities themselves - they are expressed in terms of the 2D-carrier densities $n^{\mathrm{QW}}$ and $p^{\mathrm{QW}}$ as follows: ${ }^{17,18}$

$$
f_{\mathrm{n}}=1-\exp \left(-\frac{n^{\mathrm{QW}}}{N_{\mathrm{c}}^{2 \mathrm{D}}}\right), \quad f_{\mathrm{p}}=1-\exp \left(-\frac{p^{\mathrm{QW}}}{N_{\mathrm{v}}^{2 \mathrm{D}}}\right),
$$

where $N_{\mathrm{c}, \mathrm{v}}^{2 \mathrm{D}}=m_{\mathrm{c}, \mathrm{v}}^{\mathrm{QW}} T /\left(\pi \hbar^{2}\right)$ are the $2 \mathrm{D}$ effective densities of states in the conduction and valence bands in the $\mathrm{QW}, m_{\mathrm{c}, \mathrm{v}}^{\mathrm{QW}}$ are the electron and hole effective masses in the QW, and the temperature $T$ is measured in units of energy.

We now list the parameters entering into Eqs. (1)-(5): $b$ is the thickness of the OCL, $j$ is the injection current density, $e$ is the electron charge, $N_{\mathrm{QW}}$ is the number of $\mathrm{QWs}, B_{3 \mathrm{D}}$ and $B_{2 \mathrm{D}}$ are the spontaneous radiative recombination constants for the bulk (OCL) and 2D regions (QWs) measured in units of $\mathrm{cm}^{3} / \mathrm{s}$ and $\mathrm{cm}^{2} / \mathrm{s}$, respectively, $\mathrm{v}_{\mathrm{g}}$ is the group velocity of light, $g^{\max }\left(f_{\mathrm{n}}+f_{\mathrm{p}}-1\right)$ is the modal gain provided by each of

$$
n_{1}=N_{\mathrm{c}}^{3 \mathrm{D}} \exp \left(-\frac{\Delta E_{\mathrm{c}}-\varepsilon_{\mathrm{n}}^{\mathrm{QW}}}{T}\right)
$$

$p_{1}=N_{\mathrm{v}}^{3 \mathrm{D}} \exp \left(-\frac{\Delta E_{\mathrm{v}}-\varepsilon_{\mathrm{p}}^{\mathrm{QW}}}{T}\right)$,

where $N_{\mathrm{c}, \mathrm{v}}^{3 \mathrm{D}}=2\left[m_{\mathrm{c}, \mathrm{v}}^{\mathrm{OCL}} T /\left(2 \pi \hbar^{2}\right)\right]^{3 / 2}$ are the $3 \mathrm{D}$ effective densities of states in the conduction and valence bands in the OCL, $m_{\mathrm{c}, \mathrm{v}}^{\mathrm{OCL}}$ are the electron and hole effective masses in the OCL, $\Delta E_{\mathrm{c}, \mathrm{v}}$ are the conduction and valence band offsets between the OCL and the QW, and $\varepsilon_{\mathrm{n}, \mathrm{p}}^{\mathrm{QW}}$ are the energies of the electron- and hole-sub-band edges in the QW.

Solving the set of rate equations (1)-(5) and, particularly, finding the number of photons $N$ as a function of the injection current density $j$ will allow us to calculate the lightcurrent characteristic (LCC) of the laser (the output optical power $P$ versus $j$ ),

$$
P(j)=\hbar \omega \mathrm{v}_{\mathrm{g}} \beta N(j),
$$

where $\hbar \omega$ is the photon energy.

Introducing the current density of stimulated recombination in the laser active region (i.e., in the QWs) and the internal differential quantum efficiency, ${ }^{19}$

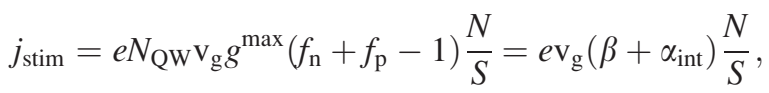

the QWs, $g^{\max }$ is the maximum value of the modal gain in each QW, $S=W L$ is the cross-section of the junction, $W$ is the lateral size of the device, $L$ is the cavity length, $\beta=$ $(1 / L) \ln (1 / R)$ is the mirror loss, $R$ is the facet reflectivity, and $\alpha_{\text {int }}$ is the internal optical loss.

We denoted the thermal escape times of electrons and holes from a QW to the OCL by $\tau_{\mathrm{n}, \mathrm{p}, \mathrm{esc}}$ and the capture velocities from the OCL into an empty (at $\left.f_{\mathrm{n}, \mathrm{p}}=0\right) \mathrm{QW}$ by $\mathrm{v}_{\mathrm{n}, \mathrm{p}, \text { capt }, 0}$. It is the capture velocity (measured in $\mathrm{cm} / \mathrm{s}$ ), which is a proper parameter describing the carrier capture into a QW. ${ }^{6-11,14-16}$ The thermal escape time and the capture velocity are related to each other (see Ref. 16 for the general expression relating $\tau_{\mathrm{n}, \mathrm{p}, \mathrm{esc}}$ and $\left.\mathrm{v}_{\mathrm{n}, \mathrm{p}, \mathrm{capt}, 0}\right)$. For undoped OCL and QW, the relation is

$$
\tau_{\mathrm{n}, \mathrm{esc}}=\frac{1}{\mathrm{v}_{\mathrm{n}, \mathrm{capt}, 0}\left(1-f_{\mathrm{n}}\right)} \frac{N_{\mathrm{c}}^{2 \mathrm{D}}}{n_{1}}, \quad \tau_{\mathrm{p}, \mathrm{esc}}=\frac{1}{\mathrm{v}_{\mathrm{p}, \mathrm{capt}, 0}\left(1-f_{\mathrm{p}}\right)} \frac{N_{\mathrm{v}}^{2 \mathrm{D}}}{p_{1}} .
$$

In Eq. (7), the quantities $n_{1}$ and $p_{1}$ are

$$
\eta_{\text {int }}=\frac{j_{\text {stim }}}{j-j_{\text {th }}}
$$

where $j_{\text {th }}$ is the threshold current density [see Eq. (A14)], we can write Eq. (9) as follows:

$$
P(j)=\frac{\hbar \omega}{e} S j_{\text {stim }}(j) \frac{\beta}{\beta+\alpha_{\mathrm{int}}}=\frac{\hbar \omega}{e} S\left(j-j_{\mathrm{th}}\right) \eta_{\mathrm{int}}(j) \frac{\beta}{\beta+\alpha_{\mathrm{int}}} .
$$

When writing the second equation in Eq. (10), we used the lasing condition (5).

\section{DISCUSSION}

In Appendix, the set of rate equations (1)-(5) is solved. The following closed-form expression is derived for $\eta_{\text {int }}$, which applies to QW lasers, quantum wire lasers, and quantum dot lasers:

$$
\eta_{\text {int }}(j)=\left\{\frac{1}{2}+\frac{1}{2}\left(\frac{1}{j_{\text {capt }, \mathrm{n}, \mathrm{th}}}+\frac{1}{j_{\text {capt }, \mathrm{p}, \mathrm{th}}}\right) j_{\text {spon }, \mathrm{th}}^{\mathrm{OCL}}+\sqrt{\left[\frac{1}{2}+\frac{1}{2}\left(\frac{1}{j_{\text {capt } \mathrm{n}, \mathrm{th}}}+\frac{1}{j_{\text {capt }, \mathrm{p}, \mathrm{th}}}\right) j_{\text {spon, th }}^{\mathrm{OCL}}\right]^{2}+\frac{j_{\text {spon,th }}^{\mathrm{OCL}}}{j_{\text {capt }, \mathrm{n}, \mathrm{th}} j_{\text {capt }, \mathrm{p}, \mathrm{th}}}\left(j-j_{\text {th }}\right)}\right\}^{-1}
$$


where $j_{\text {spon,th }}^{\mathrm{OCL}}$ is the spontaneous recombination current density in the OCL at the lasing threshold [see Eq. (A15)] and $j_{\text {capt,n,th }}, j_{\text {capt,p,th }}$ are the current densities of electron and hole capture from the OCL into a quantum-confined active region at the lasing threshold.

Equation (13) can be rewritten as follows:

$$
\eta_{\text {int }}(j)=\left[\frac{1}{2}+\frac{j_{\text {spon }, \text { th }}^{\text {OCL }}}{\left\langle j_{\text {capt } \text { th }}\right\rangle_{\text {harmon }}}+\sqrt{\left(\frac{1}{2}+\frac{j_{\text {spon }, \text { th }}^{\text {OCL }}}{\left\langle j_{\text {capt }, \text { th }}\right\rangle_{\text {harmon }}}\right)^{2}+\frac{j_{\text {spon }, \text { th }}^{\text {OCL }}}{\left\langle j_{\text {capt th }}\right\rangle_{\text {geom }}} \frac{j-j_{\text {th }}}{\left\langle j_{\text {capt th }}\right\rangle_{\text {geom }}}}\right]^{-1},
$$

where we introduced the harmonic and geometric means of the current densities of electron and hole capture at the lasing threshold,

$$
\begin{aligned}
& \frac{1}{\left\langle j_{\text {capt th }}\right\rangle_{\text {harmon }}}=\frac{1}{2}\left(\frac{1}{j_{\text {capt }, \mathrm{n}, \mathrm{th}}}+\frac{1}{j_{\text {capt } \mathrm{p}, \mathrm{th}}}\right), \\
& \left\langle j_{\text {capt,th }}\right\rangle_{\text {geom }}=\sqrt{j_{\text {capt }, \mathrm{n}, \text { th }} j_{\text {capt }, \mathrm{p}, \text { th }}} .
\end{aligned}
$$

If the electron and hole parameters (capture velocities, effective masses, conduction and valence band offsets) were the same, the rate equations (2) and (4) would become identical to the rate equations (1) and (3), respectively, and our "five rate equations" model would naturally reduce to the "three rate equations" model of Refs. 14 and 15. The internal differential quantum efficiency would be given by Eq. (14) in which both $\left\langle j_{\text {capt,th }}\right\rangle_{\text {harmon }}$ and $\left\langle j_{\text {capt,th }}\right\rangle_{\text {geom }}$ would simply turn into the capture current density at the lasing threshold, which would be the same for electrons and holes. Hence, we see that, in the expression for $\eta_{\text {int }}$ in the "five rate equations model," the asymmetry between the electron and hole capture is effectively taken into account by the harmonic and geometric means of the current densities of electron and hole capture at the lasing threshold. In case of significant asymmetry, these mean quantities $\left\langle j_{\text {capt,th }}\right\rangle_{\text {harmon }}$ and $\left\langle j_{\text {capt,th }}\right\rangle_{\text {geom }}$ differ considerably from either $j_{\text {capt, } \mathrm{n} \text {, th }}$ and $j_{\text {capt, } \mathrm{p} \text {, th }}$.

For QW lasers, using Eqs. (A25) and (A26) for $j_{\text {capt, n, th }}$ and $j_{\text {capt,p,th }}$, we can rewrite Eq. (13) as follows:

$$
\begin{aligned}
\eta_{\text {int }}(j)= & \left(\frac{1}{2}+\frac{1}{2} \frac{b B_{3 \mathrm{D}}}{N_{\mathrm{QW}}}\left[\frac{p_{\mathrm{th}}^{\mathrm{OCL}}}{\mathrm{v}_{\mathrm{capt}, \mathrm{n}, 0}\left(1-f_{\mathrm{n}}\right)}+\frac{n_{\mathrm{th}}^{\mathrm{OCL}}}{\mathrm{v}_{\mathrm{capt}, \mathrm{p}, 0}\left(1-f_{\mathrm{p}}\right)}\right]\right. \\
& +\sqrt{\left\{\frac{1}{2}+\frac{1}{2} \frac{b B_{3 \mathrm{D}}}{N_{\mathrm{QW}}}\left[\frac{p_{\mathrm{th}}^{\mathrm{OCL}}}{\mathrm{v}_{\mathrm{capt}, \mathrm{n}, 0}\left(1-f_{\mathrm{n}}\right)}+\frac{n_{\mathrm{th}}^{\mathrm{OCL}}}{\mathrm{v}_{\mathrm{capt}, \mathrm{p}, 0}\left(1-f_{\mathrm{p}}\right)}\right]\right\}^{2}+\frac{b B_{3 \mathrm{D}}}{e N_{\mathrm{QW}}^{2} \mathrm{v}_{\text {capt }, \mathrm{n}, 0} \mathrm{v}_{\text {capt }, \mathrm{p}, 0}\left(1-f_{\mathrm{n}}\right)\left(1-f_{\mathrm{p}}\right)}\left(j-j_{\mathrm{th}}\right)},
\end{aligned}
$$

where $n_{\mathrm{th}}^{\mathrm{OCL}}$ and $p_{\mathrm{th}}^{\mathrm{OCL}}$ are the threshold values of $n^{\mathrm{OCL}}$ and $p^{\mathrm{OCL}}$, which are given by Eqs. (A12) and (A13), respectively.

Using Eq. (17), we calculate the LCC of the laser [Eq. (12)], the free-electron and -hole densities and spontaneous recombination current density in the OCL [Eqs. (A27), (A28), and (A2)], the stimulated recombination current density [from Eq. (11)], and the number of photons in the lasing mode [from Eq. (10)].

For illustration of our theoretical model, we use an InGaAs/GaAs/AlGaAs heterostructure laser with a broadened waveguide. ${ }^{20}$ The QW material is $\operatorname{In}_{0.28} \mathrm{Ga}_{0.72} \mathrm{As}$ and the QW thickness is $80 \AA$. The lasing wavelength is $1.044 \mu \mathrm{m}$. The materials of the waveguide region (OCL) and cladding layers are $\mathrm{GaAs}$ and $\mathrm{Al}_{0.3} \mathrm{Ga}_{0.7} \mathrm{As}$, respectively. The thickness of the OCL $b=1.7 \mu \mathrm{m}$. The other parameters of the structure are: $L=1.5 \mathrm{~mm}, R=0.32, \beta=7.6 \mathrm{~cm}^{-1}$, $\alpha_{\text {int }}=1 \mathrm{~cm}^{-1}$, and $T=300 \mathrm{~K}$. We consider structures with a single QW (Figs. 1-3) and multiple QWs (Figs. 4 and 5).

Figure 1 shows the free-electron (a) and -hole (b) densities in the OCL against injection current density $j$ above the lasing threshold for several values of the electron and hole capture velocities $\mathrm{v}_{\mathrm{n}, \mathrm{p}, \text { capt }, 0}$. Both $n^{\mathrm{OCL}}$ and $p^{\mathrm{OCL}}$ increase considerably with increasing $j$. As seen from Eqs. (A27) and (A28) [or Eqs. (A9) and (A10)], this increase is due to the fact that the electron and hole capture from the OCL into the QW is not instantaneous-in the case of instantaneous capture $\left(\mathrm{v}_{\mathrm{n}, \mathrm{p}, \text { capt }, 0} \rightarrow \infty\right.$ and $\left.j_{\text {capt, n,p,th }} \rightarrow \infty\right), n^{\mathrm{OCL}}$ and $p^{\mathrm{OCL}}$ would remain pinned at their threshold values $n_{\mathrm{th}}^{\mathrm{OCL}}$ and $p_{\text {th }}^{\mathrm{OCL}}$. The comparison of curve 1 with curve 3 and curve 2 with curve 4 in Fig. 1(a) [and also curve 1 with curve 2 and curve 3 with curve 4 in Fig. 1(b)] shows that, at a given $j$, the higher is the capture velocity for carriers of one type (at a fixed capture velocity for carriers of the opposite type), the lower is the density of carriers of that type-naturally, the faster is the carrier capture into a low-dimensional active region, the fewer carriers are accumulated in a bulk reservoir region (OCL). At the same time, and notably, the density of carriers of the opposite type becomes higher-compare curve 1 with curve 2 and curve 3 with curve 4 in Fig. 1(a) [and also curve 1 with curve 3 and curve 2 with curve 4 in 
(a)

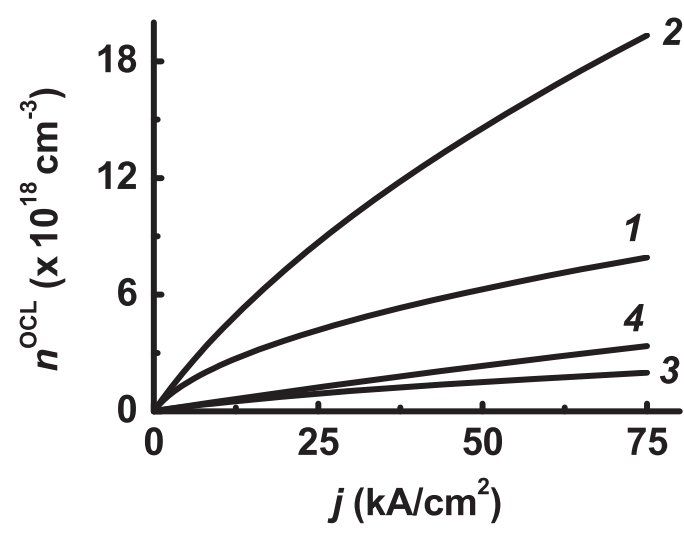

(b)

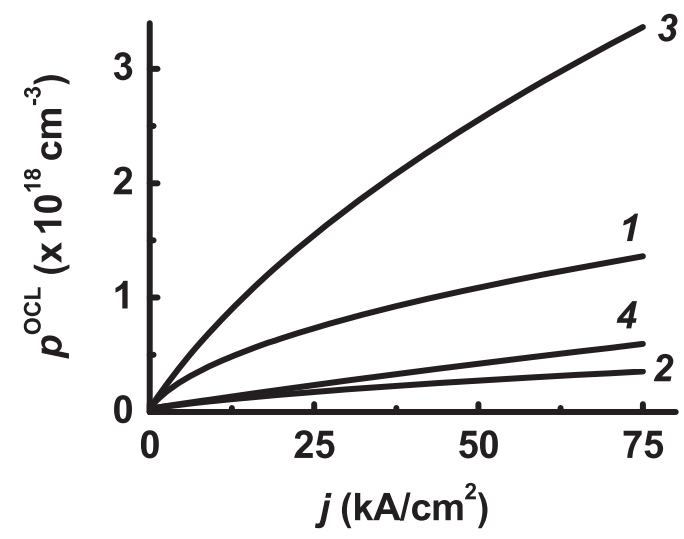

FIG. 1. Free-electron (a) and -hole (b) densities in the OCL vs. injection current density above the lasing threshold. Figs. 1-3 are for laser structures with a single QW. In Figs. 1-3, curves 1-4 correspond to the following values of the electron and hole capture velocities: $1-\mathrm{v}_{\mathrm{n}, \mathrm{capt}, 0}=10^{5} \mathrm{~cm} / \mathrm{s}, \mathrm{v}_{\mathrm{p}, \mathrm{capt}, 0}$ $=10^{5} \mathrm{~cm} / \mathrm{s} ; \quad 2-\mathrm{v}_{\mathrm{n}, \text { capt }, 0}=10^{5} \mathrm{~cm} / \mathrm{s}, \quad \mathrm{v}_{\mathrm{p}, \text { capt }, 0}=10^{6} \mathrm{~cm} / \mathrm{s} ; \quad 3-\mathrm{v}_{\mathrm{n}, \text { capt }, 0}$ $=10^{6} \mathrm{~cm} / \mathrm{s}, \mathrm{v}_{\mathrm{p}, \text { capt }, 0}=10^{5} \mathrm{~cm} / \mathrm{s} ; 4-\mathrm{v}_{\mathrm{n}, \text { capt }, 0}=10^{6} \mathrm{~cm} / \mathrm{s}, \mathrm{v}_{\mathrm{p}, \text { capt }, 0}=10^{6} \mathrm{~cm} / \mathrm{s}$. The threshold current density values for curves $1-4$ are: $1-j_{\mathrm{th}}=82.9 \mathrm{~A} / \mathrm{cm}^{2}$; $2-j_{\mathrm{th}}=81.4 \mathrm{~A} / \mathrm{cm}^{2} ; 3-j_{\mathrm{th}}=76.1 \mathrm{~A} / \mathrm{cm}^{2} ; 4-j_{\mathrm{th}}=75.7 \mathrm{~A} / \mathrm{cm}^{2}$.

Fig. 1(b)]. These tendencies can be easily understood from Eqs. (A27) and (A28). For the sake of definiteness, let us consider increasing $\mathrm{v}_{\mathrm{n} \text {, capt }, 0}$ at a fixed $\mathrm{v}_{\mathrm{p} \text {, capt }, 0}$. Both $\eta_{\text {int }}$ [see Fig. 3(a)] and $j_{\text {capt, } n, \text { th }}$ increase with $\mathrm{v}_{\mathrm{n}, \text { capt }, 0}$ but $j_{\text {capt, } \mathrm{n}, \mathrm{th}}$ increases faster, since it is directly proportional to $\mathrm{v}_{\mathrm{n} \text {, capt }, 0}$ [see Eq. (A25)]. Hence, the ratio $\eta_{\text {int }} / j_{\text {capt, n,th }}$ in Eq. (A27) decreases and so does $n^{\text {OCL }}$ [if $\mathrm{v}_{\mathrm{n} \text {, capt }, 0}$ is not very low, the change in $n_{\mathrm{th}}^{\mathrm{OCL}}$ and $j_{\text {th }}$ with $\mathrm{v}_{\mathrm{n} \text {, capt }, 0}$ can be neglected in Eq. (A27)—see the $j_{\text {th }}$ values in the caption to Fig. 1]. In contrast to $\eta_{\text {int }} / j_{\text {capt, } \mathrm{n}, \mathrm{th}}$, since $j_{\text {capt, } \mathrm{p}, \mathrm{th}}$ is fixed, the ratio $\eta_{\text {int }} / j_{\text {capt }, \mathrm{p}, \mathrm{th}}$ in Eq. (A28) increases with $\mathrm{v}_{\mathrm{n} \text {, capt }, 0}$ and so does $p^{\mathrm{OCL}}$.

Figure 2 shows the current densities of spontaneous recombination in the OCL (a) and stimulated recombination in the QW (b) against injection current density $j$ for several values of $\mathrm{v}_{\mathrm{n}, \mathrm{p} \text {, capt }, 0}$. Both $j_{\text {spon }}^{\mathrm{OCL}}$ and $j_{\text {stim }}$ increase with $j$. Since the spontaneous radiative recombination is bimolecular $\left[j_{\mathrm{spon}}^{\mathrm{OCL}}\right.$ is proportional to the product of $n^{\mathrm{OCL}}$ and $p^{\mathrm{OCL}}$ - see Eq. (A2)], $j_{\text {spon }}^{\mathrm{OCL}}$ increases superlinearly [Fig. 2(a)]. As seen from Eq. (A1), the sum $j_{\text {spon }}^{\mathrm{OCL}}+j_{\text {stim }}$ is equal to $j-j_{\text {spon }}^{\mathrm{QW}}$, i.e., is linear in $j$ (as discussed in Appendix, the current density $j_{\text {spon }}^{\mathrm{QW}}$ of spontaneous recombination in the $\mathrm{QW}$ is pinned at its threshold value and does not change with $j$ ). Hence, it follows that $j_{\text {stim }}$ should be sublinear in $j$ [Fig. 2(b)]. The faster either the electron or hole capture into the $\mathrm{QW}$, the lower is $j_{\text {spon }}^{\mathrm{OCL}}$ and, (a)

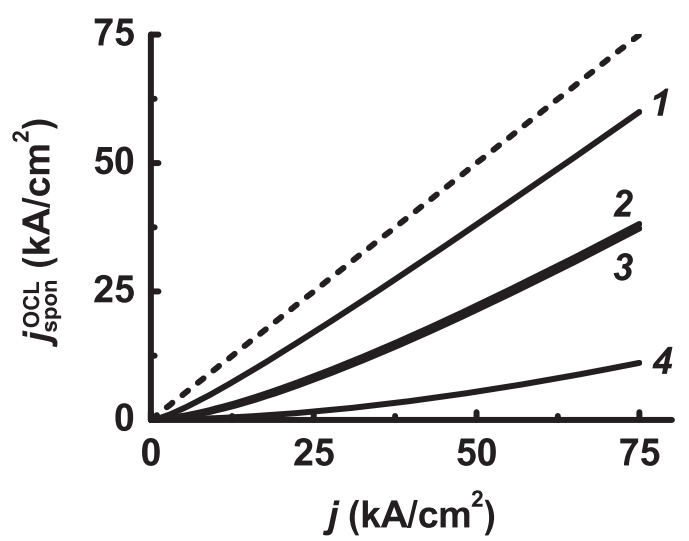

(b)

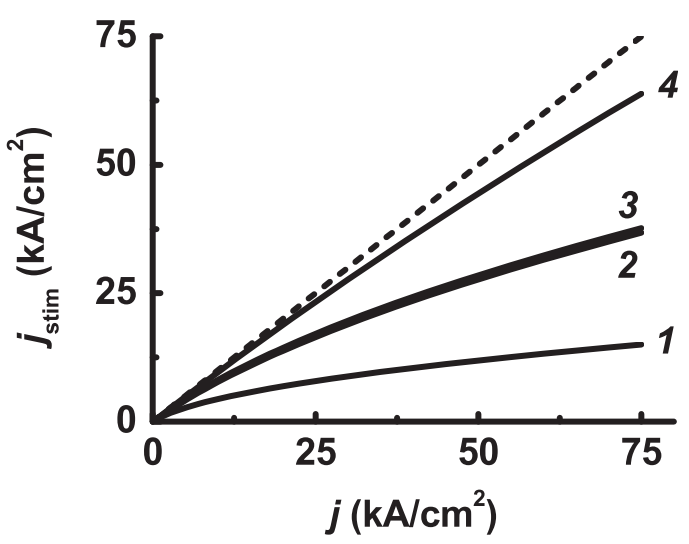

FIG. 2. Current densities of spontaneous recombination in the OCL (a) and stimulated recombination in the QW (b) vs. injection current density. The dashed lines show the injection current density itself.

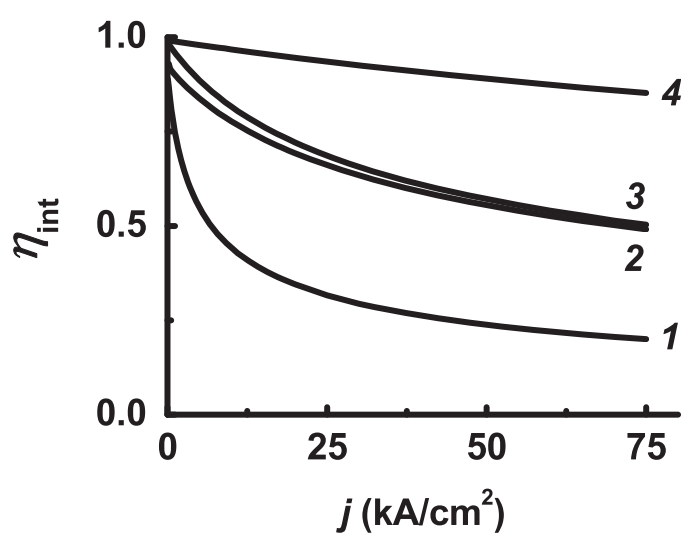

(b)

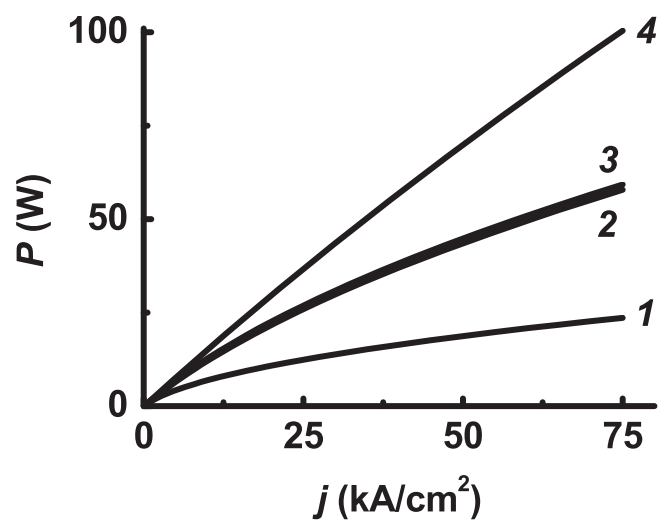

FIG. 3. Internal differential quantum efficiency (a) and output optical power (b) vs. injection current density. 


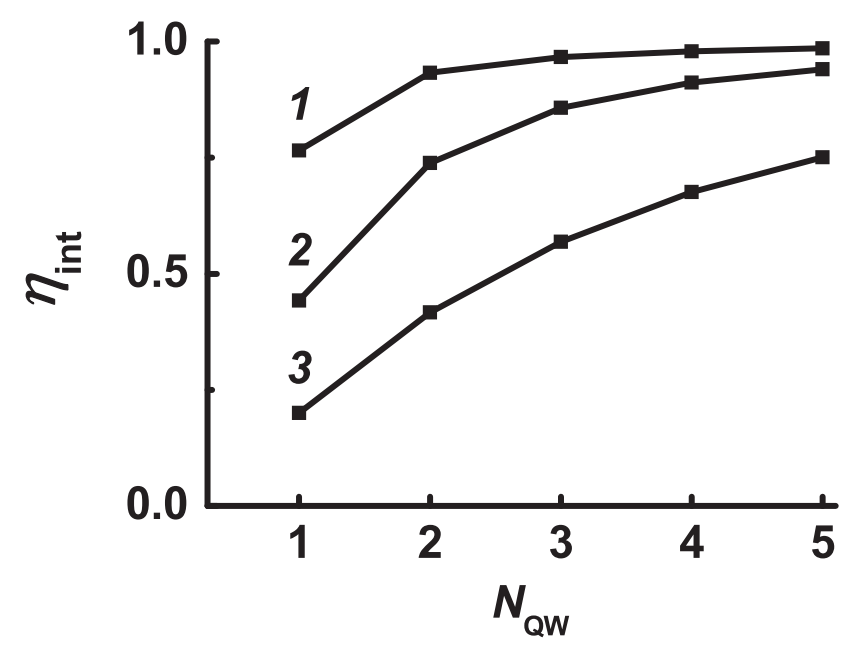

FIG. 4. Internal quantum efficiency vs. number of QWs for different values of the injection current density: $1-j=1 \mathrm{kA} / \mathrm{cm}^{2} ; 2-j=10 \mathrm{kA} / \mathrm{cm}^{2} ; 3-j$ $=75 \mathrm{kA} / \mathrm{cm}^{2}$. In Figs. 4 and $5, \mathrm{v}_{\mathrm{n}, \text { capt }, 0}=10^{5} \mathrm{~cm} / \mathrm{s}$ and $\mathrm{v}_{\mathrm{p}, \text { capt }, 0}=10^{5} \mathrm{~cm} / \mathrm{s}$.

consequently, the higher is $j_{\text {stim }}$. In the ideal case of instantaneous capture of both electrons and holes, $j$ spon would remain pinned at its threshold value $j_{\text {spon, th }}^{\text {OCL }}$, hence $j_{\text {stim }}$ would be equal to $j-j_{\text {spon }}^{\mathrm{QW}}-j_{\text {spon, th }}^{\mathrm{OCL}}$, i.e., to $j-j_{\text {th }}$ [see Eq. (A14)], and the internal differential quantum efficiency [see Eq. (11)] would be equal to unity.

Figure 3 shows the internal quantum efficiency (a) and the output optical power (b) of the laser against injection current density for several values of $v_{n, p, c a p t, 0}$. As it follows from Eq. (11) and seen from Fig. 3(a), $\eta_{\text {int }}$ decreases with $j$-since $j_{\text {stim }}$ increases sublinearly with $j$ [Fig. 2(b)], the ratio of $j_{\text {stim }}$ to $j-j_{\text {th }}$ defining $\eta_{\text {int }}$ is a decreasing function of $j$. As is clear from our discussion, the decrease of $\eta_{\text {int }}$ is due to electron and hole capture delay into the QW and bimolecular character of spontaneous recombination in the OCL. Due to sublinearity of $j_{\text {stim }}$, so is the LCC of the laser [Fig. 3(b)]. Except for the case of fast capture of both electrons and holes into the QW [curves 4 in Figs. 3(a) and 3(b)], the drop of $\eta_{\text {int }}$ with $j$ and the sublinearity of the LCC are significant.

For the case of slow capture of both electrons and holes into individual QWs, Fig. 4 shows the internal quantum

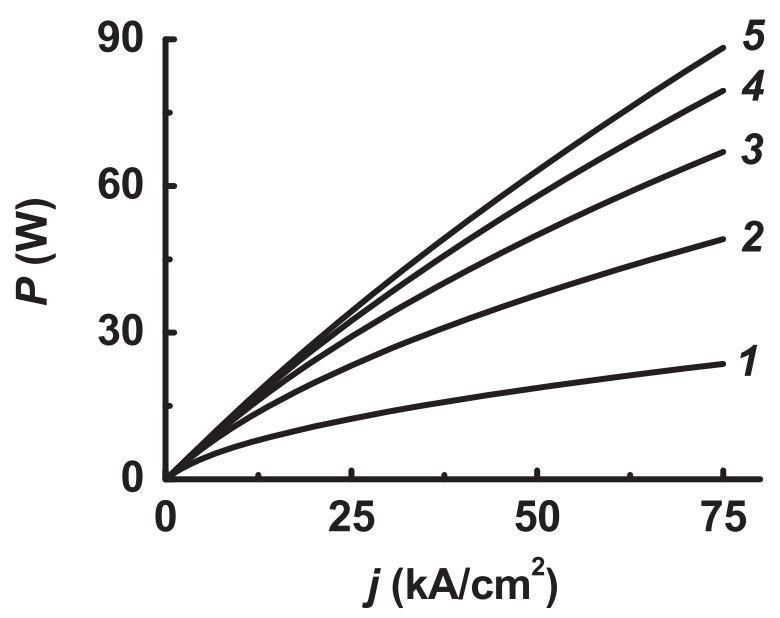

FIG. 5. Light-current characteristics of laser structures with multiple QWs: $1-N_{\mathrm{QW}}=1 ; 2-N_{\mathrm{QW}}=2 ; 3-N_{\mathrm{QW}}=3 ; 4-N_{\mathrm{QW}}=4 ; 5-N_{\mathrm{QW}}=5$. efficiency against number of QWs and Fig. 5 shows the LCC of laser structures with multiple QWs.

As seen from Eq. (17) and Fig. 4, $\eta_{\text {int }}$ becomes higher with increasing number of QWs. This is because both the electron and hole capture current densities are directly proportional to $N_{\mathrm{QW}}$ [see Eqs. (A16) and (A17)] and hence increasing $N_{\mathrm{QW}}$ acts similarly to increasing both the electron and hole capture velocities $\mathrm{v}_{\mathrm{n}, \mathrm{p} \text {, capt, } 0}$ into individual QWs.

Due to increase in $\eta_{\text {int }}$, the output optical power increases and the LCC becomes considerably more linear with increasing number of QWs (Fig. 5). Hence, if the carrier capture into individual QWs is not fast, the use of multiple QWs can effectively enhance the internal quantum efficiency and linearity of the LCC of the laser.

\section{CONCLUSIONS}

We have developed a comprehensive analytical model for the operating characteristics of semiconductor lasers with a low-dimensional active region. Particular emphasis has been given to the effect of capture delay of both electrons and holes from a bulk OCL into a quantum-confined active region and an extended set of rate equations have been used. We have derived closed-form expressions for the electron and hole densities, current densities of spontaneous and stimulated recombination, internal quantum efficiency, and output optical power as explicit functions of the injection current and parameters of a laser structure. Due to either electron or hole capture delay, the internal efficiency decreases with increasing injection current above the lasing threshold thus causing sublinearity of the LCC of a laser. As an illustration of our model, we have considered QW lasers.

\section{ACKNOWLEDGMENTS}

L.V.A. acknowledges the U.S. Army Research Office (Grant No. W911NF-13-1-0445) for support of this work.

\section{APPENDIX: DERIVATION OF SOLUTIONS TO RATE EQUATIONS}

We derive here closed-form expressions for the solutions of the set of rate equations (1)-(5).

Using Eq. (3) or (4) in, respectively, Eq. (1) or (2), we can present the injection current density as a sum of the current densities of spontaneous recombination in the OCL and QWs and stimulated recombination in the QWs,

$$
j=j_{\text {spon }}^{\mathrm{OCL}}+j_{\mathrm{spon}}^{\mathrm{QW}}+j_{\mathrm{stim}}
$$

where

$$
\begin{gathered}
j_{\text {spon }}^{\mathrm{OCL}}=e b B_{3 \mathrm{D}} n^{\mathrm{OCL}} p^{\mathrm{OCL}}, \\
j_{\text {spon }}^{\mathrm{QW}}=e N_{\mathrm{QW}} B_{2 \mathrm{D}} n^{\mathrm{QW}} p^{\mathrm{QW}},
\end{gathered}
$$

and $j_{\text {stim }}$ is given by Eq. (10). 
Assuming that (i) the internal optical loss $\alpha_{\text {int }}$ does not depend on the free-carrier densities $n^{\mathrm{OCL}}$ and $p^{\mathrm{OCL}}$ in the OCL and (ii) charge neutrality holds in each QW $\left(n^{\mathrm{QW}}\right.$ $=p^{\mathrm{QW}}$ ) at the lasing threshold, we obtain from the lasing condition (5) that the electron and hole densities in the QWs do not depend on the injection current density and hence remain equal to each other also above the lasing threshold,

$$
n^{\mathrm{QW}}=p^{\mathrm{QW}}=\operatorname{const}(j) .
$$

In view of pinning the 2D-carrier densities, the spontaneous recombination current density in the QWs is also pinned above the lasing threshold,

$$
j_{\text {spon }}^{\mathrm{QW}}=e N_{\mathrm{QW}} B_{2 \mathrm{D}}\left(n^{\mathrm{QW}}\right)^{2}=\operatorname{const}(j) .
$$

With Eqs. (6) and (A4), we have from Eq. (5) the following equation for finding $n^{\mathrm{QW}}$ :

$$
\begin{aligned}
& N_{\mathrm{QW}} g^{\max }\left[1-\exp \left(-\frac{n^{\mathrm{QW}}}{N_{\mathrm{c}}^{2 \mathrm{D}}}\right)-\exp \left(-\frac{m_{\mathrm{c}}^{\mathrm{QW}}}{m_{\mathrm{v}}^{\mathrm{QW}}} \frac{n^{\mathrm{QW}}}{N_{\mathrm{c}}^{2 \mathrm{D}}}\right)\right] \\
& =\beta+\alpha_{\mathrm{int}} .
\end{aligned}
$$

With Eqs. (3) and (4), we can express $n^{\mathrm{OCL}}$ and $p^{\mathrm{OCL}}$ in terms of the 2D-carrier density in the QWs $n^{\mathrm{QW}}$ and the photon number $N$ as follows:

$$
\begin{aligned}
n^{\mathrm{OCL}}= & \frac{1}{\mathrm{v}_{\mathrm{n}, \text { capt }, 0}\left(1-f_{\mathrm{n}}\right)} \\
& \times\left[\frac{n^{\mathrm{QW}}}{\tau_{\mathrm{n}, \mathrm{esc}}}+B_{2 \mathrm{D}}\left(n^{\mathrm{QW}}\right)^{2}+\mathrm{v}_{\mathrm{g}} g^{\max }\left(f_{\mathrm{n}}+f_{\mathrm{p}}-1\right) \frac{N}{S}\right], \\
p^{\mathrm{OCL}}= & \frac{1}{\mathrm{v}_{\mathrm{p}, \text { capt }, 0}\left(1-f_{\mathrm{p}}\right)} \\
& \times\left[\frac{n^{\mathrm{QW}}}{\tau_{\mathrm{p}, \mathrm{esc}}}+B_{2 \mathrm{D}}\left(n^{\mathrm{QW}}\right)^{2}+\mathrm{v}_{\mathrm{g}} g^{\max }\left(f_{\mathrm{n}}+f_{\mathrm{p}}-1\right) \frac{N}{S}\right],
\end{aligned}
$$

or, using Eq. (7),

$$
\begin{aligned}
n^{\mathrm{OCL}}= & n_{1} \frac{n^{\mathrm{QW}}}{N_{\mathrm{c}}^{2 \mathrm{D}}}+\frac{1}{\mathrm{v}_{\mathrm{n}, \text { capt }, 0}\left(1-f_{\mathrm{n}}\right)} \\
& \times\left[B_{2 \mathrm{D}}\left(n^{\mathrm{QW}}\right)^{2}+\mathrm{v}_{\mathrm{g}} g^{\max }\left(f_{\mathrm{n}}+f_{\mathrm{p}}-1\right) \frac{N}{S}\right], \\
p^{\mathrm{OCL}}= & p_{1} \frac{n^{\mathrm{QW}}}{N_{\mathrm{v}}^{2 \mathrm{D}}}+\frac{1}{\mathrm{v}_{\mathrm{p}, \text { capt }, 0}\left(1-f_{\mathrm{p}}\right)} \\
& \times\left[B_{2 \mathrm{D}}\left(n^{\mathrm{QW}}\right)^{2}+\mathrm{v}_{\mathrm{g}} g^{\max }\left(f_{\mathrm{n}}+f_{\mathrm{p}}-1\right) \frac{N}{S}\right] .
\end{aligned}
$$

As seen from Eqs. (A9) and (A10), the following linear relationship between $n^{\mathrm{OCL}}$ and $p^{\mathrm{OCL}}$ holds:

$$
\begin{aligned}
& \left(n^{\mathrm{OCL}}-n_{1} \frac{n^{\mathrm{QW}}}{N_{\mathrm{c}}^{2 \mathrm{D}}}\right) \mathrm{v}_{\mathrm{n}, \text { capt }, 0}\left(1-f_{\mathrm{n}}\right) \\
& =\left(p^{\mathrm{OCL}}-p_{1} \frac{n^{\mathrm{QW}}}{N_{\mathrm{v}}^{2 \mathrm{D}}}\right) \mathrm{v}_{\mathrm{p}, \text { capt }, 0}\left(1-f_{\mathrm{p}}\right) .
\end{aligned}
$$

The threshold values of $n^{\mathrm{OCL}}$ and $p^{\mathrm{OCL}}$ are obtained by putting the number of photons $N=0$ in Eqs. (A9) and (A10),

$$
\begin{aligned}
& n_{\mathrm{th}}^{\mathrm{OCL}}=n_{1} \frac{n^{\mathrm{QW}}}{N_{\mathrm{c}}^{2 \mathrm{D}}}+\frac{1}{\mathrm{v}_{\mathrm{n}, \text { capt }, 0}\left(1-f_{\mathrm{n}}\right)} B_{2 \mathrm{D}}\left(n^{\mathrm{QW}}\right)^{2}, \\
& p_{\mathrm{th}}^{\mathrm{OCL}}=p_{1} \frac{n^{\mathrm{QW}}}{N_{\mathrm{v}}^{2 \mathrm{D}}}+\frac{1}{\mathrm{v}_{\mathrm{p}, \text { capt }, 0}\left(1-f_{\mathrm{p}}\right)} B_{2 \mathrm{D}}\left(n^{\mathrm{QW}}\right)^{2} .
\end{aligned}
$$

The threshold current density is

$$
j_{\mathrm{th}}=j_{\mathrm{spon}, \mathrm{th}}^{\mathrm{OCL}}+j_{\mathrm{spon}}^{\mathrm{QW}}
$$

where the spontaneous recombination current density in the OCL at the lasing threshold is

$$
j_{\mathrm{spon}, \mathrm{th}}^{\mathrm{OCL}}=e b B_{3 \mathrm{D}} n_{\mathrm{th}}^{\mathrm{OCL}} p_{\mathrm{th}}^{\mathrm{OCL}} .
$$

Let us introduce the current densities of electron and hole capture from the OCL into the QWs and escape from the QWs to the OCL,

$$
\begin{aligned}
& j_{\text {capt }, \mathrm{n}}=e N_{\mathrm{QW}} \mathrm{v}_{\mathrm{n}, \text { capt }} n^{\mathrm{OCL}}=e N_{\mathrm{QW}} \mathrm{V}_{\mathrm{n}, \text { capt }, 0}\left(1-f_{\mathrm{n}}\right) n^{\mathrm{OCL}}, \\
& j_{\text {capt }, \mathrm{p}}=e N_{\mathrm{QW} \mathrm{v}_{\mathrm{p}, \text { capt }}} p^{\mathrm{OCL}}=e N_{\mathrm{QW} \mathrm{V}_{\mathrm{p}, \text { capt }, 0}\left(1-f_{\mathrm{p}}\right) p^{\mathrm{OCL}},}
\end{aligned}
$$

In Eqs. (A16) and (A17), $\mathrm{v}_{\mathrm{n}, \text { capt }}$ and $\mathrm{v}_{\mathrm{p}, \mathrm{capt}}$ are the capture velocities, which take into account the QW filling by electrons and holes and are related to the capture velocities $\mathrm{v}_{\mathrm{n}, \text { capt }, 0}$ and $\mathrm{v}_{\mathrm{p}, \text { capt }, 0}$ into an unoccupied QW as follows:

$$
\mathrm{v}_{\mathrm{n}, \text { capt }}=\mathrm{v}_{\mathrm{n}, \text { capt }, 0}\left(1-f_{\mathrm{n}}\right), \quad \mathrm{v}_{\mathrm{p}, \text { capt }}=\mathrm{v}_{\mathrm{p}, \mathrm{capt}, 0}\left(1-f_{\mathrm{p}}\right) .
$$

Since the 2D-carrier densities are pinned [see Eq. (A4)], the escape current densities [Eqs. (A18) and (A19)] are also pinned above the lasing threshold.

From Eqs. (3) and (4), we have

$$
\begin{aligned}
& j_{\text {capt }, \mathrm{n}}=j_{\mathrm{esc}, \mathrm{n}}+j_{\text {spon }}^{\mathrm{QW}}+j_{\text {stim }}, \\
& j_{\text {capt }, \mathrm{p}}=j_{\mathrm{esc}, \mathrm{p}}+j_{\text {spon }}^{\mathrm{QW}}+j_{\text {stim }} .
\end{aligned}
$$


Since $j_{\text {esc,n }}, j_{\text {esc,p }}$, and $j_{\text {spon }}^{\mathrm{QW}}$ do not change with increasing $j$ above the lasing threshold, we can write Eqs. (A21) and (A22) in the form

$$
\begin{aligned}
& j_{\text {capt }, \mathrm{n}}(j)=j_{\text {capt }, \mathrm{n}, \mathrm{th}}+j_{\text {stim }}(j), \\
& j_{\text {capt }, \mathrm{p}}(j)=j_{\text {capt }, \mathrm{p}, \mathrm{th}}+j_{\text {stim }}(j),
\end{aligned}
$$

where the capture current densities at the lasing threshold are

$$
\begin{aligned}
& j_{\text {capt }, \mathrm{n}, \mathrm{th}}=e N_{\mathrm{QW}} \mathrm{v}_{\mathrm{n}, \mathrm{capt}} n_{\mathrm{th}}^{\mathrm{OCL}}=j_{\mathrm{esc}, \mathrm{n}}+j_{\mathrm{spon}}^{\mathrm{QW}}, \\
& j_{\text {capt } \mathrm{p}, \mathrm{th}}=e N_{\mathrm{QW}} \mathrm{v}_{\mathrm{p}, \mathrm{capt}} p_{\mathrm{th}}^{\mathrm{OCL}}=j_{\mathrm{esc}, \mathrm{p}}+j_{\text {spon }}^{\mathrm{QW}} .
\end{aligned}
$$

Using Eqs. (A16), (A17) and Eqs. (A23)-(A26), we can write the free carrier densities as follows:

$$
\begin{aligned}
& n^{\mathrm{OCL}}(j)=\frac{j_{\text {capt }, \mathrm{n}}(j)}{e N_{\mathrm{QW}} \mathrm{V}_{\mathrm{n}, \mathrm{capt}}}=n_{\mathrm{th}}^{\mathrm{OCL}} \frac{j_{\text {capt }, \mathrm{n}}(j)}{j_{\text {capt }, \mathrm{n}, \mathrm{th}}} \\
& =n_{\mathrm{th}}^{\mathrm{OCL}}\left[1+\frac{j_{\text {stim }}(j)}{j_{\text {capt }, \mathrm{n}, \mathrm{th}}}\right]=n_{\mathrm{th}}^{\mathrm{OCL}}\left[1+\frac{j-j_{\mathrm{th}}}{j_{\text {capt } \mathrm{n}, \mathrm{th}}} \eta_{\mathrm{int}}(j)\right], \\
& p^{\mathrm{OCL}}(j)=\frac{j_{\text {capt }, \mathrm{p}}(j)}{e N_{\mathrm{QW}} \mathrm{v}_{\mathrm{p}, \text { capt }}}=p_{\text {th }}^{\mathrm{OCL}} \frac{j_{\text {capt }, \mathrm{p}}(j)}{j_{\text {capt }, \mathrm{p}, \text { th }}} \\
& =p_{\mathrm{th}}^{\mathrm{OCL}}\left[1+\frac{j_{\text {stim }}(j)}{j_{\text {capt }, \mathrm{p}, \mathrm{th}}}\right]=p_{\mathrm{th}}^{\mathrm{OCL}}\left[1+\frac{j-j_{\mathrm{th}}}{j_{\text {capt }, \mathrm{p}, \mathrm{th}}} \eta_{\text {int }}(j)\right] \text {. }
\end{aligned}
$$

We used Eq. (11) in the last equations in Eqs. (A27) and (A28).

Using Eq. (A14), we can write Eq. (A1) in the form

$$
j-j_{\text {th }}=\left[j_{\text {spon }}^{\mathrm{OCL}}(j)-j_{\text {spon }, \mathrm{Oh}}^{\mathrm{OCL}}\right]+j_{\mathrm{stim}}(j)
$$

or

$$
\frac{j-j_{\mathrm{th}}}{j_{\mathrm{spon}, \mathrm{th}}^{\mathrm{OCL}}}=\left[\frac{n^{\mathrm{OCL}}(j)}{n_{\mathrm{th}}^{\mathrm{OCL}}} \frac{p^{\mathrm{OCL}}(j)}{p_{\mathrm{th}}^{\mathrm{OCL}}}-1\right]+\frac{j_{\mathrm{stim}}(j)}{j_{\mathrm{spon}, \mathrm{OC}}^{\mathrm{OCL}}} .
$$

Using Eqs. (A27), (A28), and (11) in Eq. (A30), we obtain the following quadratic equation in the internal differential quantum efficiency $\eta_{\text {int }}(j)$ :

$$
\begin{aligned}
& \frac{j_{\text {spon,th }}^{\text {OCL }}}{j_{\text {capt }, \mathrm{n}, \text { th }} j_{\text {capt }, \mathrm{p}, \text { th }}}\left(j-j_{\text {th }}\right) \eta_{\text {int }}^{2}(j) \\
& \quad+\left[1+\left(\frac{1}{j_{\text {capt }, \mathrm{n}, \mathrm{th}}}+\frac{1}{j_{\text {capt }, \mathrm{p}, \mathrm{th}}}\right) j_{\text {spon,th }}^{\mathrm{OCL}}\right] \eta_{\text {int }}(j)-1=0 .
\end{aligned}
$$

The solution of Eq. (A31) is given by Eq. (13). With Eq. (13), we easily calculate the LCC of the laser [Eq. (12)], the free carrier densities in the OCL [Eqs. (A27) and (A28)], the spontaneous recombination current density in the OCL [Eq. (A2)], the stimulated recombination current density [from Eq. (11)], and the number of photons in the lasing mode [from Eq. (10)].

${ }^{1}$ P. S. Zory, Jr., Quantum Well Lasers (Academic, Boston, 1993).

${ }^{2}$ E. Kapon, Semiconductor Lasers: Fundamentals (Academic, New York, 1999).

${ }^{3}$ S. V. Kozyrev and A. Y. Shik, Sov. Phys. Semicond. 19, 1024 (1985).

${ }^{4}$ J. A. Brum and G. Bastard, Phys. Rev. B 33, 1420 (1986).

${ }^{5}$ N. Debbar, D. Biswas, and P. Bhattacharya, Phys. Rev. B 40, 1058 (1989).

${ }^{6}$ I. N. Yassievich, K. Schmalz, and M. Beer, Semicond. Sci. Technol. 9, 1763 (1994).

${ }^{7}$ E. Rosencher, B. Vinter, F. Luc, L. Thibaudeau, P. Bois, and J. Nagle, IEEE Trans. Quantum Electron. 30, 2875 (1994).

${ }^{8}$ C. Y. Tsai, Y. H. Lo, R. M. Spencer, and L. F. Eastman, IEEE J. Sel. Top. Quantum Electron. 1, 316 (1995).

${ }^{9}$ S. A. Solov'ev, I. N. Yassievich, and V. M. Chistyakov, Semiconductors 29, 654 (1995).

${ }^{10}$ R. A. Suris, NATO ASI Ser. E 323, 197 (1996).

${ }^{11}$ A. Dargys and J. Kundrotas, Semicond. Sci. Technol. 13, 1258 (1998).

${ }^{12}$ I. Esquivias, S. Weisser, B. Romero, J. D. Ralston, and J. Rosenzweig, IEEE Photon. Technol. Lett. 8, 1294 (1996).

${ }^{13}$ K. Kalna and M. Mosko, Phys. Rev. B 54, 17730 (1996).

${ }^{14}$ L. V. Asryan, S. Luryi, and R. A. Suris, Appl. Phys. Lett. 81, 2154 (2002).

${ }^{15}$ L. V. Asryan, S. Luryi, and R. A. Suris, IEEE J. Quantum Electron. 39, 404 (2003).

${ }^{16}$ D.-S. Han and L. V. Asryan, Nanotechnology 21, 015201 (2010).

${ }^{17}$ L. V. Asryan and S. Luryi, Appl. Phys. Lett. 83, 5368 (2003).

${ }^{18}$ L. V. Asryan and S. Luryi, IEEE J. Quantum Electron. 40, 833 (2004).

${ }^{19}$ L. A. Coldren and S. W. Corzine, Diode Lasers and Photonic Integrated Circuits (Wiley, New York, 1995).

${ }^{20}$ A. V. Lyutetskii, K. S. Borshchev, N. A. Pikhtin, S. O. Slipchenko, Z. N. Sokolova, and I. S. Tarasov, Semiconductors 42, 104 (2008). 